\title{
ANTARCTIC ICE SHEET SURFACE OXYGEN ISOTOPE VALUES
}

\author{
By V. I. MORGAN \\ (Antarctic Division, Department of Science, Kingston, Tasmania, Australia 7150)
}

\begin{abstract}
Collected data on the mean annual surface values for $\delta^{18} \mathrm{O}$ over Antarctica have been tabulated and also presented in map form. An additional map shows contours of constant $\delta^{18} \mathrm{O}$ values.

RÉSUmé. Teneurs en isotope de l'oxygène à la surface de la calotte glaciaire antarctique. Les données recueillies sur les valeurs annuelles moyennes en surface pour la teneur en $\delta^{18} \mathrm{O}$ dans l'ensemble de l'Antarctique ont été compilées et sont également présentées sous forme cartographique. Une carte supplémentaire montre les lignes d'égales teneurs en $\delta^{18} \mathrm{O}$.
\end{abstract}

ZuSAmmenfassung. Werte für Sauerstoff-Isotope an der Oberfläche der antartischen Eisdecke. Datenmaterial über die mittleren jährlichen Oberflächenwerte von $\delta^{18} \mathrm{O}$ in Antarktika wurde tabuliert und auch in kartographischer Form dargestellt. Eine zusätzliche Karte enthält Linien konstanter $\delta^{18} \mathrm{O}-\mathrm{Werte}$.

\section{INTRODUCTION}

One of the difficulties in interpreting oxygen-isotope data from deep cores is in making allowance for the different place of deposition of the lower layers. If the direction and velocity of the ice flow is known, a first-order correction to the core data can be made by considering the variations in the present-day surface $\delta^{18} \mathrm{O}$. For more sophisticated studies, however, changes in the surface isotope ratio which will have occurred with changes to the ice sheet also have to be considered and this presents considerable difficulty.

The prominent change in the $\delta^{18} \mathrm{O}$ values found in the deep core data around 10000 years B.P. (see e.g. Johnsen and others (1972)) is rather too large to be solely due to temperature and probably also reflects a general lowering of the ice sheet surface in conjunction with a retreat of the ice margin. Both the lowering and the reduction in area will reduce the depletion of ${ }^{18} \mathrm{O}$ in the surface snow but at present it is not easy to assign magnitudes to the changes.

The main idea behind this collection and presentation of data is to give a broad-scale picture of the surface $\delta^{18} \mathrm{O}$ for comparison with core $\delta^{18} \mathrm{O}$ profiles. A second use is that future examinations of the compiled data will enable a better understanding of the variations in the depletion in ${ }^{18} \mathrm{O}$ in Antarctic precipitation in terms of the temperature, elevation, continentality, etc. of the particular location.

The experimentally observed relationship between $\delta^{18} \mathrm{O}$ and temperature arises because of the decrease in the saturation vapour pressure of water with temperature. A cooling air mass must precipitate water to avoid supersaturation and as this water will be enriched $(c .+8 \%$ in ${ }^{18} \mathrm{O}$ with respect to the vapour, the vapour will therefore become progressively depleted in ${ }^{18} \mathrm{O}$. The mechanism, complicated by non-equilibrium transfers of water vapour, is affected by the path the air mass takes for instance over land, sea, or ice, its temperature and rate of temperature change, its turbulence, and other factors most of which are climatic variables and thus presumably will have varied in the past.

It is envisaged that the data presented here in Figs 1 and 2 and Table I may be used to obtain empirical values for the fractionation processes which affect precipitation falling on different parts of Antarctica and hence can be used to adjust deep core $\delta^{18} \mathrm{O}$ data for such factors as changes in ice-sheet elevation and area. 


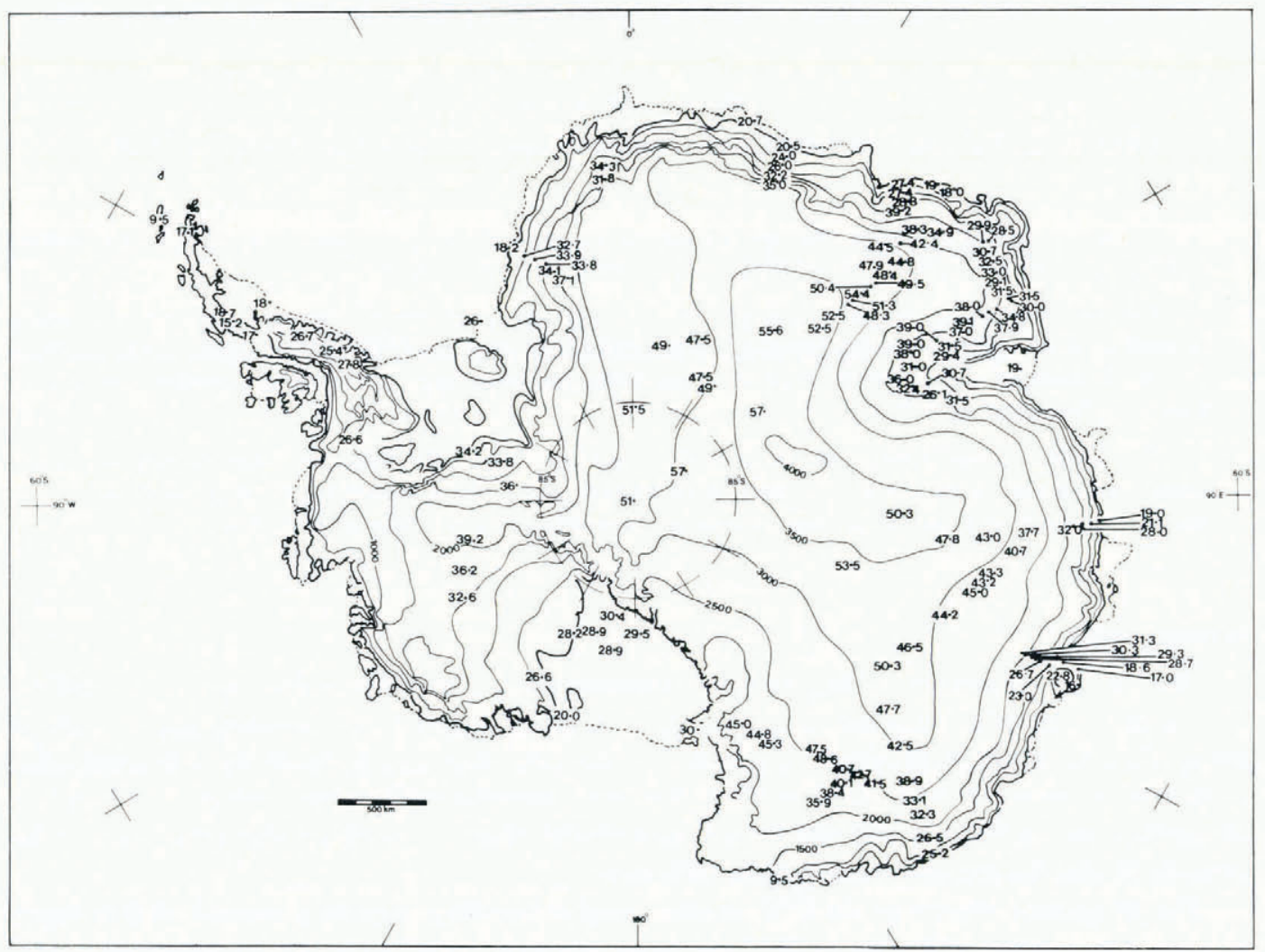

Fig. 1. Mean annual surface values of $\delta^{18} O$ in parts per thousand obtained from measurements in Antarctica.

\section{SAMPLE AND DATA COLLECTION}

The large seasonal $\delta^{18} \mathrm{O}$ variability means that some form of averaging is required to obtain a value which approximates the long-term mean. Where deep bore-hole analysis data are available, this is effectively done by averaging values from the top $50 \mathrm{~m}$ or so of core. Where only shallow cores are obtained, e.g. by a hand auger or where pit samples are used, an even number of seasons' accumulation will probably not be collected and, due to the relatively small number of seasons represented, the $\delta^{18} \mathrm{O}$ value will be biased away from the long-term mean.

We can obtain some idea of the magnitudes involved by examining a core from the Law Dome summit for which some detailed measurements have been made. (See Budd and Morgan, 1977). This location is probably representative of one of the more difficult places to sample because the high annual accumulation and the general lack of wind (for Antarctica) allows the large seasonal $\delta$ variation to be well preserved in amplitude. The accumulation is $0.6 \mathrm{Mg} \mathrm{m}^{-2} \mathrm{a}^{-1}$ and the range of isotope values is $8 \%$ at $100 \mathrm{~m}$ depth, and probably about $12 \%$ near the surface. Assuming roughly equal amounts of summer and winter precipitation, the deviation in the $\delta$ value from the mean if an exactly odd number of seasons are sampled is:

spread in $\delta$

$\overline{2 \times \text { no. of seasons sampled }}$ 


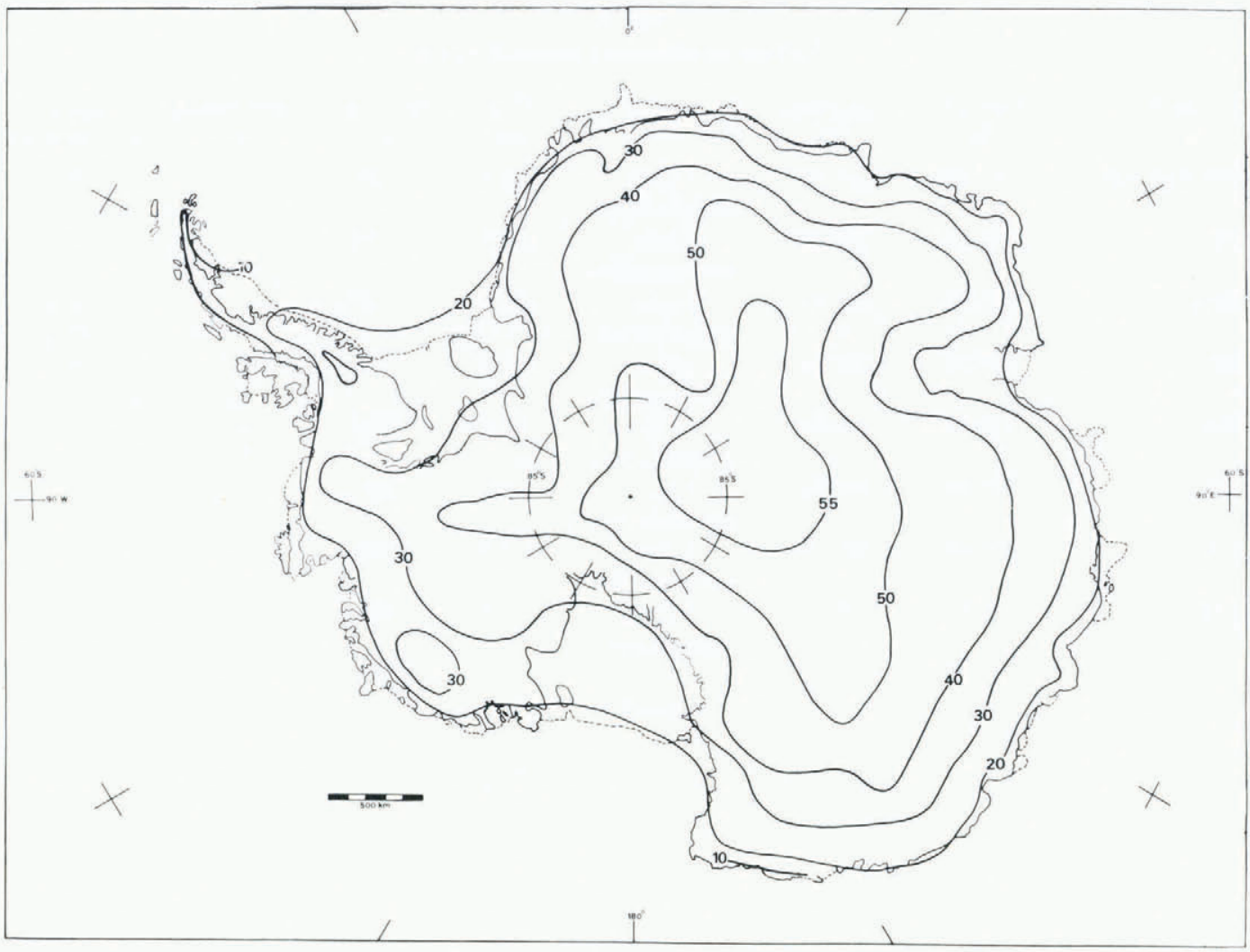

Fig. 2. Contours of constant mean annual surface values of $\delta^{18} O$ in parts per thousand derived from the point values in Figure 1.

To have a sample within $1 \%$ of the long-term in such a location requires that at least six seasons (i.e. three years) accumulation be sampled. This could cover a depth of $4 \mathrm{~m}$ in the nearcoastal areas, but will be much less inland, where the accumulation rate is much smaller.

Some of the data used may be marginal in respect of sampling depth. Where possible the depth covered by the sample is noted in the listing of data.

The measurements are given as $\delta^{18} \mathrm{O}$ with respect to SMOW, expressed as parts per mil deviation (see Craig, 1961). Where a number of measurements are closely spaced, e.g. on the Law Dome, the number of points has been reduced by averaging to give a reasonable spacing on the map (Fig. 1). The second map shows contours of constant $\delta^{18} \mathrm{O}$ values (Fig. 2).

\section{ACKNOWLEDGEMENTS}

I would like to acknowledge the work done by various A.N.A.R.E. (Australian National Antarctic Research Expedition) personnel in collecting samples while on traverse and also in particular the assistance of T. H. Jacka and J. Birch in analysing samples for $\delta^{18} \mathrm{O}$ and collating data.

$M S$. received 6 April 1981 
TABLE I

ANTARCTIC ICE SHEET SURFACE DATA

\begin{tabular}{|c|c|c|c|c|c|c|c|}
\hline Location & $\begin{array}{c}\text { Latitude } \\
\mathrm{S}\end{array}$ & $\begin{array}{c}\text { Longitude } \\
\text { E }\end{array}$ & Sample & $\begin{array}{c}\delta^{18} \mathrm{O} \\
\% \text { o }\end{array}$ & $\begin{array}{c}\text { Elevation } \\
\mathrm{m}\end{array}$ & $\begin{array}{c}\text { Temperature } \\
{ }^{\circ} \mathrm{C}\end{array}$ & $\begin{array}{c}\text { Source } \\
\text { (see key below) }\end{array}$ \\
\hline Roi Baudouin & $70^{\circ} 26^{\prime}$ & $24^{\circ} 19^{\prime}$ & $\begin{array}{c}1 \text { year } \\
\text { accumulation }\end{array}$ & -20.5 & 39 & & 1 \\
\hline PL & $69^{\circ} 54^{\prime}$ & $18^{\circ} 42^{\prime}$ & $\begin{array}{c}1 \text { year } \\
\text { accumulation }\end{array}$ & -20.7 & 16 & & 1 \\
\hline BRB & $70^{\circ} 24^{\prime}$ & $24^{\circ} 12^{\prime}$ & $\begin{array}{c}1 \text { year } \\
\text { accumulation }\end{array}$ & -21.9 & 22 & & 1 \\
\hline ED & $69^{\circ} 39^{\prime}$ & $24^{\circ} 36^{\prime}$ & $\begin{array}{c}1 \text { year } \\
\text { accumulation }\end{array}$ & -21.0 & & & 1 \\
\hline W4 & $71^{\circ} 00^{\prime}$ & $24^{\circ} 06^{\prime}$ & $\begin{array}{c}1 \text { year } \\
\text { accumulation }\end{array}$ & -24.0 & 590 & & 1 \\
\hline W7 & $71^{\circ} 27^{\prime}$ & $24^{\circ} 00^{\prime}$ & $\begin{array}{c}1 \text { year } \\
\text { accumulation }\end{array}$ & -26.0 & 900 & & 1 \\
\hline G1 & $72^{\circ} 00^{\prime}$ & $24^{\circ} 18^{\prime}$ & $\begin{array}{c}1 \text { year } \\
\text { accumulation }\end{array}$ & -32.2 & 1500 & & 1 \\
\hline BM & $72^{\circ} 36^{\prime}$ & $30^{\circ} 54^{\prime}$ & $\begin{array}{c}1 \text { year } \\
\text { accumulation }\end{array}$ & -36.2 & 2000 & & 1 \\
\hline $13 \mathrm{~A}$ & $72^{\circ} 18^{\prime}$ & $24^{\circ} 21^{\prime}$ & $\begin{array}{c}1 \text { year } \\
\text { accumulation }\end{array}$ & -35.0 & 2400 & & 1 \\
\hline & c. $84^{\circ}$ & c. $1^{\circ}$ & & -51.5 & & & 2 \\
\hline & c. $82^{\circ}$ & c. $11^{\circ}$ & & -49 & & & 2 \\
\hline & c. $81^{\circ}$ & c. $21^{\circ}$ & & -47.5 & & & 2 \\
\hline & c. $82^{\circ} 18^{\prime}$ & c. $30^{\circ}$ & & -47.5 & & & 2 \\
\hline & c. $82^{\circ} 06^{\prime}$ & c. $32^{\circ}$ & & -49 & & & 2 \\
\hline & c. $83^{\circ}$ & c. $60^{\circ}$ & & -57 & & & 2 \\
\hline $\mathrm{H} 128$ & $69^{\circ} 25^{\prime}$ & $41^{\circ} 36^{\prime}$ & 5 & -27.4 & 1359 & & 3 \\
\hline S97 & $69^{\circ} 35^{\prime}$ & $42^{\circ} 48^{\prime}$ & 5 & -28.8 & 1605 & & 3 \\
\hline Y100 & $71^{\circ} 17^{\prime}$ & $46^{\circ} 19^{\prime}$ & 2 & -42.4 & 2596 & & 3 \\
\hline Y210 & $71^{\circ} 45^{\prime}$ & $48^{\circ} 57^{\prime}$ & 2 & -44.8 & 2880 & & 3 \\
\hline $\mathrm{I} 235$ & $73^{\circ} 49^{\prime}$ & $48^{\circ} 33^{\prime}$ & 2 & -50.8 & 3200 & & 3 \\
\hline $\mathrm{I} 365$ & $74^{\circ} 55^{\prime}$ & $47^{\circ} 56^{\prime}$ & 2 & -51.3 & 3.310 & & 3 \\
\hline I 485 & $76^{\circ} 00^{\prime}$ & $48^{\circ} 06^{\prime}$ & 2 & -52.5 & 3383 & & 3 \\
\hline $\mathrm{J} 225$ & $73^{\circ} 01^{\prime}$ & $46^{\circ} 22^{\prime}$ & 2 & -47.9 & 3039 & & 3 \\
\hline J364 & $71^{\circ} 50^{\prime}$ & $44^{\circ} 45^{\prime}$ & 2 & -44.5 & 2613 & & 3 \\
\hline W280 & $70^{\circ} 13^{\prime}$ & $46^{\circ} 34^{\prime}$ & 2 & -38.3 & 2405 & & 3 \\
\hline W46 & $69^{\circ} 33^{\prime}$ & $48^{\circ} 56^{\prime}$ & 2 & -34.9 & 1958 & & 3 \\
\hline Y200 & $71^{\circ} 44^{\prime}$ & $48^{\circ} 41^{\prime}$ & shallow & -46.9 & 2870 & & 4 \\
\hline $\mathrm{I} 100$ & $72^{\circ} 38^{\prime}$ & $48^{\circ} 40^{\prime}$ & sample & -48.4 & 3026 & & 4 \\
\hline $\mathrm{I} 200$ & $73^{\circ} 30^{\prime}$ & $48^{\circ} 29^{\prime}$ & below & -49.5 & 3184 & & 4 \\
\hline I 300 & $74^{\circ} 22^{\prime}$ & $48^{\circ} 07^{\prime}$ & surface & -54.4 & 3266 & & 4 \\
\hline I 400 & $75^{\circ} 14^{\prime}$ & $47^{\circ} 58^{\prime}$ & snow & -48.3 & 3329 & & 4 \\
\hline I500 & $76^{\circ} 08^{\prime}$ & $48^{\circ} 05^{\prime}$ & & -51.3 & 3385 & & 4 \\
\hline I600 & $77^{\circ} 00^{\prime}$ & $48^{\circ} 03^{\prime}$ & & -52.5 & 3408 & & 4 \\
\hline S40 & $69^{\circ} 05^{\prime}$ & $41^{\circ} 07^{\prime}$ & & -27.4 & 1112 & & 4 \\
\hline S84 & $69^{\circ} 22^{\prime}$ & $42^{\circ} 38^{\prime}$ & & -30.9 & 1518 & & 4 \\
\hline $\mathrm{S} 122$ & $70^{\circ} 01^{\prime}$ & $43^{\circ} 07^{\prime}$ & $\downarrow$ & -39.2 & 1853 & & 4 \\
\hline Molodezhnaya & $67^{\circ} 40^{\prime}$ & $45^{\circ} 50^{\prime}$ & & -20.1 & & -10.9 & 5 \\
\hline GWAM & $67^{\circ} 35^{\prime}$ & $62^{\circ} 50^{\prime}$ & & -26.1 & c. 100 & & 6 \\
\hline $\mathrm{C} 10$ & $68^{\circ} 38^{\prime}$ & $62^{\circ} 07^{\prime}$ & 2 & -30.0 & 1790 & & 6 \\
\hline C64 & $69^{\circ} 27^{\prime}$ & $62^{\circ} 13^{\prime}$ & 2 & -34.8 & 2060 & & 6 \\
\hline C 344 & $69^{\circ} 53^{\prime}$ & $62^{\circ} 00^{\prime}$ & 2 & -37.9 & 2290 & & 6 \\
\hline
\end{tabular}


TABLE I-continued

\begin{tabular}{|c|c|c|c|c|c|c|c|}
\hline Location & $\begin{array}{l}\text { Latitude } \\
\text { S }\end{array}$ & $\begin{array}{l}\text { Longitude } \\
\text { E }\end{array}$ & Sample & $\begin{array}{c}\delta^{18} \mathrm{O} \\
\% o\end{array}$ & $\begin{array}{c}\text { Elevation } \\
\mathrm{m}\end{array}$ & $\begin{array}{c}\text { Temperature } \\
{ }^{\circ} \mathrm{C}\end{array}$ & $\begin{array}{c}\text { Source } \\
\text { (see key below) }\end{array}$ \\
\hline C 124 & $70^{\circ} 15^{\prime}$ & $62^{\circ} 12^{\prime}$ & 2 & -38.0 & 2340 & & 6 \\
\hline $\mathrm{C} 175$ & $71^{\circ} 00^{\prime}$ & $62^{\circ} 10^{\prime}$ & 2 & -39.1 & 2220 & & 6 \\
\hline C216 & $71^{\circ} 29^{\prime}$ & $62^{\circ} 51^{\prime}$ & 2 & -37.0 & 1815 & & 6 \\
\hline C 244 & $71^{\circ} 52^{\prime}$ & $63^{\circ} 26^{\prime}$ & 2 & -29.2 & 1840 & & 6 \\
\hline C271 & $72^{\circ} 14^{\prime}$ & $63^{\circ} 59^{\prime}$ & 9 & -31.48 & 1545 & & 6 \\
\hline GL1 & $72^{\circ} 31^{\prime}$ & $65^{\circ} 19^{\prime}$ & 2 & -29.39 & 1148 & & 7 \\
\hline GL2 & $72^{\circ} 16^{\prime}$ & $63^{\circ} 58^{\prime}$ & 9 & -31.48 & 1607 & & 7 \\
\hline GL3 & $72^{\circ} 48^{\prime}$ & $62^{\circ} 09^{\prime}$ & 2 & -31.56 & 1808 & & 7 \\
\hline GL5 & $73^{\circ} 43^{\prime}$ & $61^{\circ} 11^{\prime}$ & 10 & -38.96 & 2000 & & 7 \\
\hline GL6 & $74^{\circ} 03^{\prime}$ & $61^{\circ} 57^{\prime}$ & 2 & -38.04 & 1889 & & 7 \\
\hline GL7 & $74^{\circ} 14^{\prime}$ & $64^{\circ} 13^{\prime}$ & 2 & -30.97 & 1556 & & 7 \\
\hline GL8 & $74^{\circ} 59^{\prime}$ & $66^{\circ} 06^{\prime}$ & 9 & -35.95 & 1763 & & 7 \\
\hline GL9 & $74^{\circ} 45^{\prime}$ & $67^{\circ} 58^{\prime}$ & 1 & -32.39 & 1710 & & 7 \\
\hline GL10 & $74^{\circ} 04^{\prime}$ & $68^{\circ} 23^{\prime}$ & 1 & -30.24 & 1109 & & 7 \\
\hline GL11 & $73^{\circ} 44^{\prime}$ & $70^{\circ} 37^{\prime}$ & $\frac{1}{2}$ & -26.13 & 1462 & & 7 \\
\hline GLF & $72^{\circ} 57^{\prime}$ & $72^{\circ} 48^{\prime}$ & 2 & -31.5 & 1518 & & 7 \\
\hline GE2 & $68^{\circ} 39^{\prime}$ & $61^{\circ} 58^{\prime}$ & 4 & -31.53 & 1862 & & 8 \\
\hline GE3 & $68^{\circ} 39^{\prime}$ & $60^{\circ} 33^{\prime}$ & 4 & -32.02 & 1878 & & 8 \\
\hline GE4 & $68^{\circ} 36^{\prime}$ & $59^{\circ} 21^{\prime}$ & 2 & -29.10 & 1921 & & 8 \\
\hline GE5 & $68^{\circ} 31^{\prime}$ & $57^{\circ} 52^{\prime}$ & 4 & -33.01 & 2203 & & 8 \\
\hline GE6 & $68^{\circ} 24^{\prime}$ & $56^{\circ} 30^{\prime}$ & 3 & -32.48 & 2054 & & 8 \\
\hline GE7 & $68^{\circ} 17^{\prime}$ & $55^{\circ} 01^{\prime}$ & 4 & -30.66 & 2004 & & 8 \\
\hline GE8 & $68^{\circ} 01^{\prime}$ & $53^{\circ} 52^{\prime}$ & 3 & -28.51 & 2133 & & 8 \\
\hline GE9 & $68^{\circ} 16^{\prime}$ & $53^{\circ} 32^{\prime}$ & 3 & -29.93 & 2199 & & 8 \\
\hline Gl (Amery) & $69^{\circ} 28^{\prime}$ & $71^{\circ} 25^{\prime}$ & c. 50 & -19 & 64 & -20.1 & 9 \\
\hline Plateau & $79^{\circ} 15^{\prime}$ & $40^{\circ} 30^{\prime}$ & & -55 & & & 10 \\
\hline $\begin{array}{l}\text { Pole of relative } \\
\text { inaccessibility }\end{array}$ & $82^{\circ} 07^{\prime}$ & $55^{\circ} 02^{\prime}$ & & -57 & & & 10 \\
\hline GM01 & $67^{\circ} 01^{\prime}$ & $93^{\circ} 18^{\prime}$ & 1 & -21.13 & 914 & & 11 \\
\hline GM02 & $67^{\circ} 26^{\prime}$ & $93^{\circ} 23^{\prime}$ & 1 & -27.96 & 1455 & & 11 \\
\hline GM03 & $67^{\circ} 52^{\prime}$ & $93^{\circ} 45^{\prime}$ & 1 & -31.95 & 1758 & & 11 \\
\hline GM07 & $70^{\circ} 27^{\prime}$ & $97^{\circ} 51^{\prime}$ & $5-10$ & -40.67 & 2760 & -39.0 & 11 \\
\hline GM10 & $71^{\circ} 38^{\prime}$ & $101^{\circ} 50^{\prime}$ & $5-10$ & -43.23 & 2979 & -47.5 & 11 \\
\hline GM13 & $73^{\circ} 14^{\prime}$ & $110^{\circ} 27^{\prime}$ & $5-10$ & -44.16 & 2963 & -52.9 & 11 \\
\hline GM14 & $72^{\circ} 11^{\prime}$ & $105^{\circ} 23^{\prime}$ & 5 & -44.97 & 2899 & & 11 \\
\hline GM15 & $71^{\circ} 54^{\prime}$ & $103^{\circ} 19^{\prime}$ & 5 & -43.32 & 2988 & & 11 \\
\hline GM20 & $73^{\circ} 48^{\prime}$ & $114^{\circ} 37^{\prime}$ & 5 & -46.48 & 3085 & & 11 \\
\hline Dome C & $74^{\circ} 30^{\prime}$ & $123^{\circ} 10^{\prime}$ & c. 100 & -50.3 & 3240 & -50.3 & 12 \\
\hline Mirny & $66^{\circ} 33^{\prime}$ & $93^{\circ} 01^{\prime}$ & & -19.0 & & -12.4 & 5 \\
\hline Pionerskaya & $69^{\circ} 44^{\prime}$ & $\left.95^{\circ} 31^{\prime}\right)$ & & -37.7 & 2740 & -37.4 & 13 \\
\hline Vostok 1 & $72^{\circ} 08^{\prime}$ & $\left.96^{\circ} 36^{\prime}\right\}$ & $\begin{array}{l}10 \text { years } \\
\text { accumulation }\end{array}$ & -42.8 & 3250 & -47.4 & 13 \\
\hline Komsomolskaya & $74^{\circ} 05^{\prime}$ & $97^{\circ} 29^{\prime}$ & accumulation & -47.8 & 3500 & -53.9 & 13 \\
\hline Vostok & $78^{\circ} 27^{\prime}$ & $106^{\circ} 48^{\prime}$ & $60-200$ & -53.5 & 3490 & -57.3 & 5 \\
\hline Watershed & $75^{\circ} 46^{\prime}$ & $93^{\circ} 49^{\prime}$ & & -50.3 & 3730 & -57.7 & 13 \\
\hline $\mathrm{BO} 03$ & $66^{\circ} 47^{\prime}$ & $\left.112^{\circ} 44^{\prime}\right)$ & & -22.84 & 1354 & & 14 \\
\hline $\mathrm{BO} 17$ & $67^{\circ} 25^{\prime}$ & $112^{\circ} 01^{\prime}$ & & -22.24 & 830 & & 14 \\
\hline BO18 & $67^{\circ} 31^{\prime}$ & $111^{\circ} 59^{\prime}$ & & -24.68 & 822 & & 14 \\
\hline BO19 & $67^{\circ} 36^{\prime}$ & $\left.111^{\circ} 58^{\prime}\right\}$ & $<10$ & -22.24 & 1001 & & 14 \\
\hline $\mathrm{BO} 22$ & $67^{\circ} 47^{\prime}$ & $111^{\circ} 58^{\prime}$ & & -24.73 & 1209 & & 14 \\
\hline $\mathrm{BO} 24$ & $67^{\circ} 58^{\prime}$ & $111^{\circ} 57^{\prime}$ & & -27.26 & 1365 & & 14 \\
\hline $\mathrm{BO} 25$ & $68^{\circ} 06^{\prime}$ & $\left.111^{\circ} 55^{\prime}\right)$ & & -26.12 & 1488 & & 14 \\
\hline
\end{tabular}


TABLE I-continued

\begin{tabular}{|c|c|c|c|c|c|c|c|}
\hline Location & $\begin{array}{l}\text { Latitude } \\
\text { S }\end{array}$ & $\begin{array}{c}\text { Longitude } \\
\text { E }\end{array}$ & Sample & $\begin{array}{c}\delta^{18} \mathrm{O} \\
\% \text { o }\end{array}$ & $\begin{array}{c}\text { Elevation } \\
\mathrm{m}\end{array}$ & $\begin{array}{c}\text { Temperature } \\
{ }^{\circ} \mathrm{C}\end{array}$ & $\begin{array}{c}\text { Source } \\
\text { (see key below) }\end{array}$ \\
\hline $\mathrm{BO} 27$ & $68^{\circ} 21^{\prime}$ & $\left.112^{\circ} 01^{\prime}\right)$ & & -28.24 & 1606 & & 14 \\
\hline $\mathrm{BO} 28$ & $68^{\circ} 24^{\prime}$ & $112^{\circ} 03^{\prime}$ & & -29.10 & 1626 & & 14 \\
\hline BO29 & $68^{\circ} 31^{\prime}$ & $112^{\circ} 04^{\prime}$ & & -28.11 & 1702 & & 14 \\
\hline $\mathrm{BO} 30$ & $68^{\circ} 33^{\prime}$ & $112^{\circ} 01^{\prime}$ & & -30.46 & 1709 & & 14 \\
\hline $\mathrm{BO} 31$ & $68^{\circ} 38^{\prime}$ & $\left.111^{\circ} 50^{\prime}\right\}$ & $<10$ & -28.97 & 1779 & & 14 \\
\hline $\mathrm{BO} 32$ & $68^{\circ} 43^{\prime}$ & $111^{\circ} 51^{\prime}$ & & -29.54 & 1837 & & 14 \\
\hline $\mathrm{BO} 33$ & $68^{\circ} 52^{\prime}$ & $111^{\circ} 50^{\prime}$ & & -30.54 & 1902 & & 14 \\
\hline $\mathrm{AO} 34$ & $68^{\circ} 56^{\prime}$ & $112^{\circ} 03^{\prime}$ & & -30.15 & 1918 & & 14 \\
\hline $\mathrm{BO} 35$ & $69^{\circ} 03^{\prime}$ & $111^{\circ} 52^{\prime}$ & & -31.29 & 1962 & & 14 \\
\hline A & $66^{\circ} 08^{\prime}$ & $110^{\circ} 57^{\prime}$ & & -16.7 & 283 & -10.1 & 15 \\
\hline $\mathrm{F}$ & $66^{\circ} 09^{\prime}$ & $111^{\circ} 00^{\prime}$ & 40 & -17.6 & 360 & -12.4 & 15 \\
\hline $\mathrm{P}$ & $66^{\circ} 14^{\prime}$ & $111^{\circ} 14^{\prime}$ & c. 30 & -18.9 & 620 & -14.3 & 15 \\
\hline B & $66^{\circ} 18^{\prime}$ & $111^{\circ} 27^{\prime}$ & c. 63 & -19.7 & 780 & -15.7 & 15 \\
\hline Q & $66^{\circ} 23^{\prime}$ & $111^{\circ} 44^{\prime}$ & c. 40 & -20.8 & 930 & -19.0 & 15 \\
\hline D & $66^{\circ} 44^{\prime}$ & $112^{\circ} 50^{\prime}$ & 30 & -22.8 & 1390 & -21.3 & 15 \\
\hline J & $65^{\circ} 56^{\prime}$ & $113^{\circ} 09^{\prime}$ & c. 40 & -17.1 & 379 & -11.0 & 15 \\
\hline $\begin{array}{l}\text { Dumont } \\
\text { d'Urville }\end{array}$ & $66^{\circ} 40^{\prime}$ & $140^{\circ} 01^{\prime}$ & $\begin{array}{l}10 \text { years } \\
\text { accumulation }\end{array}$ & -16.9 & 40 & -11.2 & 13 \\
\hline D18 & $66^{\circ} 45^{\prime}$ & $139^{\circ} 40^{\prime}$ & 10 & -20 & 460 & -16.3 & 16 \\
\hline D41 & $66^{\circ} 50^{\prime}$ & $139^{\circ} 19^{\prime}$ & 10 & -22 & 975 & -19.8 & 16 \\
\hline D45 & & & 10 & -26 & 1410 & -24.0 & 16 \\
\hline D52 & & & 10 & -30.5 & 1850 & -28.8 & 16 \\
\hline D59 & & & 10 & -32.5 & 2220 & -34.3 & 16 \\
\hline D62 & & & 10 & -34.5 & 2290 & -37.9 & 16 \\
\hline D80 & $70^{\circ} 01^{\prime}$ & $134^{\circ} 49^{\prime}$ & 10 & -39 & 2430 & -42.1 & 16 \\
\hline D 100 & & & 10 & -42.5 & 2810 & -46.5 & 16 \\
\hline D113 & & & 10 & -47.7 & 2990 & -46.5 & 16 \\
\hline D120 & $73^{\circ} 04^{\prime}$ & $128^{\circ} 44^{\prime}$ & 10 & -47.9 & 3010 & -53.5 & 16 \\
\hline A 3 & $66^{\circ} 42^{\prime}$ & $\left.139^{\circ} 55^{\prime}\right)$ & & -19.7 & 220 & -14.5 & 13 \\
\hline A5 & $66^{\circ} 43^{\prime}$ & $139^{\circ} 55^{\prime}$ & & -20.0 & 230 & -15.6 & 13 \\
\hline A 14 & $66^{\circ} 44^{\prime}$ & $139^{\circ} 42^{\prime}$ & & -20.2 & 405 & -16.1 & 13 \\
\hline A 17 & $66^{\circ} 45^{\prime}$ & $139^{\circ} 36^{\prime}$ & & -19.5 & 470 & -17.8 & 13 \\
\hline A 28 & $66^{\circ} 49^{\prime}$ & $139^{\circ} 22^{\prime}$ & & -19.7 & 680 & -19.3 & 13 \\
\hline $\mathrm{A} 34(\mathrm{~B} 10)$ & $66^{\circ} 50^{\prime}$ & $139^{\circ} 15^{\prime}$ & & -19.3 & 790 & -19.8 & 13 \\
\hline B11 & $66^{\circ} 55^{\prime}$ & $139^{\circ} 16^{\prime}$ & & -22.1 & 985 & -22 & 13 \\
\hline B 13 & $67^{\circ} 05^{\prime}$ & $139^{\circ} 16^{\prime}$ & & -21.8 & 1210 & -24.2 & 13 \\
\hline B16 & $67^{\circ} 22^{\prime}$ & $139^{\circ} 17^{\prime}$ & & -25.8 & 1480 & -26.9 & 13 \\
\hline B17 & $67^{\circ} 26^{\prime}$ & $139^{\circ} 17^{\prime}$ & & -28.2 & 1530 & -27.4 & 13 \\
\hline B 18 & $67^{\circ} 31^{\prime}$ & $139^{\circ} 18^{\prime}$ & 10 years & -27.0 & 1610 & -28.3 & 13 \\
\hline B19 & $67^{\circ} 37^{\prime}$ & $139^{\circ} 13^{\prime}$ & accumulation & -26.7 & 1670 & -28.9 & 13 \\
\hline B22 & $67^{\circ} 55^{\prime}$ & $139^{\circ} 19^{\prime}$ & & -29.1 & 1830 & -30.4 & 13 \\
\hline B61 & $71^{\circ} 07^{\prime}$ & $139^{\circ} 15^{\prime}$ & & -41.3 & 2510 & -43.9 & 13 \\
\hline 500 & $78^{\circ} 02^{\prime}$ & $154^{\circ} 06^{\prime}$ & & -44.8 & 2280 & -43.6 & 13 \\
\hline 504 & $77^{\circ} 02^{\prime}$ & $152^{\circ} 19^{\prime}$ & & -44.6 & 2440 & -45 & 13 \\
\hline 507 & $76^{\circ} 27^{\prime}$ & $150^{\circ} 24^{\prime}$ & & -45.7 & 2480 & -47.3 & 13 \\
\hline 516 & $74^{\circ} 27^{\prime}$ & $144^{\circ} 15^{\prime}$ & & -47.3 & 2590 & -47.1 & 13 \\
\hline 519 & $73^{\circ} 44^{\prime}$ & $143^{\circ} 28^{\prime}$ & & -48.5 & 2540 & -46.9 & 13 \\
\hline 521 & $73^{\circ} 07^{\prime}$ & $142^{\circ} 30^{\prime}$ & & -43.3 & 2515 & -46.5 & 13 \\
\hline 524 & $72^{\circ} 28^{\prime}$ & $141^{\circ} 21^{\prime}$ & & -40.5 & 2500 & -44 & 13 \\
\hline 527 & $71^{\circ} 50^{\prime}$ & $140^{\circ} 15^{\prime}$ & & -42.5 & 2470 & -44.2 & 13 \\
\hline 536 & $72^{\circ} 06^{\prime}$ & $\left.143^{\circ} 11^{\prime}\right]$ & & -39.8 & 2360 & -44.3 & 13 \\
\hline
\end{tabular}


TABLE I-continued

\begin{tabular}{|c|c|c|c|c|c|c|c|}
\hline Location & $\begin{array}{c}\text { Latitude } \\
\text { S }\end{array}$ & $\begin{array}{c}\text { Longitude } \\
\text { E }\end{array}$ & Sample & $\begin{array}{c}\delta^{18} \mathrm{O} \\
\% \mathrm{o}\end{array}$ & $\begin{array}{c}\text { Elevation } \\
\mathrm{m}\end{array}$ & $\begin{array}{c}\text { Temperature } \\
{ }^{\circ} \mathrm{C}\end{array}$ & $\begin{array}{c}\text { Source } \\
\text { (see key below) }\end{array}$ \\
\hline 540 & $72^{\circ} 07^{\prime}$ & $\left.145^{\circ} 53^{\prime}\right\}$ & 10 years & -38.2 & 2290 & -42.3 & 13 \\
\hline 544 & $72^{\circ} 08^{\prime}$ & $\left.148^{\circ} 12^{\prime}\right\}$ & accumulation & -35.7 & 2220 & -41.5 & 13 \\
\hline Leningradskaya & $69^{\circ} 30^{\prime}$ & $159^{\circ} 25^{\prime}$ & & -9.5 & & -4.1 & 5 \\
\hline NZCS 16 & $77^{\circ} 51^{\prime}$ & $166^{\circ} 45^{\prime}$ & $\begin{array}{c}10 \text { years } \\
\text { accumulation }\end{array}$ & -30 & & -18 & 13 \\
\hline South Pole & & W & & -51 & 2800 & -50.7 & 17 \\
\hline Little America & $78^{\circ} 05^{\prime}$ & $162^{\circ} 28^{\prime}$ & $5-20$ & -19.7 & 43 & -24.2 & 18 \\
\hline Eights & $75^{\circ} 14^{\prime}$ & $77^{\circ} 10^{\prime}$ & & -26.6 & 452 & & 19 \\
\hline A & $80^{\circ} 55^{\prime}$ & $\left.111^{\circ} 05^{\prime}\right)$ & & -36.5 & 1700 & -29.5 & 13 \\
\hline B & $81^{\circ} 35^{\prime}$ & $102^{\circ} 40^{\prime}$ & & -38.9 & 2200 & -33.3 & 13 \\
\hline $\mathrm{C}$ & $82^{\circ} 05^{\prime}$ & $89^{\circ} 30^{\prime}$ & & -41.0 & 1800 & -33.4 & 13 \\
\hline $\mathrm{D}$ & $80^{\circ} 32^{\prime}$ & $80^{\circ} 55^{\prime}$ & 10 years & -35.2 & 950 & -27.3 & 13 \\
\hline $\mathrm{E}$ & $82^{\circ} 48^{\prime}$ & $70^{\circ} 40^{\prime}$ & accumulation & -33.5 & 500 & -27.7 & 13 \\
\hline $\mathrm{F}$ & $80^{\circ} 57^{\prime}$ & $71^{\circ} 20^{\prime}$ & & -34.8 & 150 & -29.4 & 13 \\
\hline G & $83^{\circ} 54^{\prime}$ & $78^{\circ} 30^{\prime}$ & & -35.8 & 1150 & -32.6 & 13 \\
\hline K (New Byrd) & $80^{\circ} 01^{\prime}$ & $119^{\circ} 32^{\prime}$ & & -35.8 & 1525 & -28.2 & 13 \\
\hline Byrd & & & & -32.6 & 1530 & -28.2 & 20 \\
\hline Bellingshausen & $62^{\circ} 15^{\prime}$ & $58^{\circ} 50^{\prime}$ & & -9.5 & & -3.8 & 5 \\
\hline 1 & $64^{\circ} 05^{\prime}$ & $59^{\circ} 35^{\prime}$ & 10 & -17.13 & 1806 & -14.8 & 21 \\
\hline 2 & $66^{\circ} 25^{\prime}$ & $64^{\circ} 57^{\prime}$ & 10 & -19.08 & 1937 & -15.9 & 21 \\
\hline 3 & $67^{\circ} 32^{\prime}$ & $66^{\circ} 00^{\prime}$ & 10 & -18.70 & 1750 & -16.5 & 21 \\
\hline 4 & $67^{\circ} 46^{\prime}$ & $68^{\circ} 55^{\prime}$ & 10 & -14.92 & 377 & -7.7 & 21 \\
\hline 5 & $68^{\circ} 11^{\prime}$ & $67^{\circ} 00^{\prime}$ & 10 & -15.25 & 380 & -8.1 & 21 \\
\hline 6 & $68^{\circ} 46^{\prime}$ & $60^{\circ} 56^{\prime}$ & 10 & -17.90 & 290 & -14.9 & 21 \\
\hline 7 & $69^{\circ} 30^{\prime}$ & $66^{\circ} 16^{\prime}$ & 10 & -16.80 & 870 & -12.9 & 21 \\
\hline 8 & $70^{\circ} 01^{\prime}$ & $64^{\circ} 29^{\prime}$ & 10 & -27.13 & 2131 & -21.0 & 21 \\
\hline 9 & $70^{\circ} 37^{\prime}$ & $60^{\circ} 44^{\prime}$ & 10 & -19.61 & 396 & -17.5 & 21 \\
\hline 10 & $71^{\circ} 07^{\prime}$ & $62^{\circ} 20^{\prime}$ & 10 & -25.94 & 1050 & -16.6 & 21 \\
\hline 11 & $71^{\circ} 14^{\prime}$ & $63^{\circ} 22^{\prime}$ & 10 & -26.68 & 1752 & -22.1 & 21 \\
\hline 12 & $70^{\circ} 50^{\prime}$ & $64^{\circ} 27^{\prime}$ & 10 & -26.48 & 1987 & -21.4 & 21 \\
\hline 13 & $71^{\circ} 15^{\prime}$ & $64^{\circ} 30^{\prime}$ & 10 & -25.43 & 2010 & -23.7 & 21 \\
\hline 14 & $71^{\circ} 18^{\prime}$ & $67^{\circ} 29^{\prime}$ & 10 & -13.19 & 290 & -8.4 & 21 \\
\hline 15 & $71^{\circ} 23^{\prime}$ & $65^{\circ} 30^{\prime}$ & 10 & -23.90 & 1547 & -20.0 & 21 \\
\hline 16 & $71^{\circ} 29^{\prime}$ & $66^{\circ} 58^{\prime}$ & 10 & -18.00 & 946 & -13.1 & 21 \\
\hline 17 & $70^{\circ} 00^{\prime}$ & $75^{\circ} 20^{\prime}$ & 10 & -12.60 & 595 & -12.1 & 21 \\
\hline 18 & $71^{\circ} 42^{\prime}$ & $64^{\circ} 05^{\prime}$ & 10 & -24.72 & 1886 & -20.4 & 21 \\
\hline 20 & $72^{\circ} 30^{\prime}$ & $72^{\circ} 50^{\prime}$ & 10 & -16.55 & 488 & -13.3 & 21 \\
\hline 21 & $72^{\circ} 47^{\prime}$ & $64^{\circ} 30^{\prime}$ & 10 & -25.40 & 1797 & -21.8 & 21 \\
\hline 22 & $72^{\circ} 50^{\prime}$ & $64^{\circ} 30^{\prime}$ & 10 & -16.69 & 539 & -13.3 & 21 \\
\hline 23 & $73^{\circ} 42^{\prime}$ & $64^{\circ} 47^{\prime}$ & 10 & -27.84 & 2007 & -23.8 & 21 \\
\hline 24 & $72^{\circ} 12^{\prime}$ & $60^{\circ} 20^{\prime}$ & 10 & -21.42 & 130 & -17.6 & 21 \\
\hline 25 & $70^{\circ} 53^{\prime}$ & $64^{\circ} 57^{\prime}$ & 10 & -25.77 & 1835 & -20.2 & 21 \\
\hline Ellsworth & $77^{\circ} 43^{\prime}$ & $41^{\circ} 02^{\prime}$ & New Snow & -26.6 & 20 & -22.3 & 18 \\
\hline Halley Bay & $75^{\circ} 30^{\prime}$ & $26^{\circ} 40^{\prime}$ & & -18.2 & & & 2 \\
\hline Traverse & & & & -32.7 & & & 2 \\
\hline inland of & to & to & & -33.9 & & & 2 \\
\hline Halley Bay & to & 10 & & -33.8 & & & 2 \\
\hline & & & & -34.1 & & & 2 \\
\hline & $78^{\circ} 36^{\prime}$ & $19^{\circ} 36^{\prime}$ & & -37.1 & & c. -32 & 2 \\
\hline
\end{tabular}


Data sources and references: 1. Gonfiantini and others (1963). 2. Personal communication from C. Lorius. 3. Watanabe (1977). 4. Katō (1977). 5. Gordiyenko and others (1976). 6. A.N.A.R.E. Traverses; $\delta^{18} \mathrm{O}$ by Antarctic Division. 7. Allison (1979); $\delta^{18} \mathrm{O}$ by Antarctic Division. 8. Morgan and Jacka (1981); $\delta^{18} \mathrm{O}$ by Antarctic Division. 9. Morgan (1972). 10. Dansgaard and others (1973). 11. Young (1979); $\delta^{18} \mathrm{O}$ by Antarctic Division. 12. Lorius and others (1979). 13. Lorius and others, (1969); using relation $\delta^{18} \mathrm{O}=(\delta \mathrm{D}-11) / 8.1$. 14. Budd and Young $(1979) ; \delta^{18} \mathrm{O}$ by Antarctic Division. 15. Budd and Morgan (1977). 16. Lorius and Merlivat (1977). 17. Epstein and others (1965). 18. Dansgaard and others (1977). 19. Epstein and Sharp (1967). 20. Johnsen and others (1972). 21. Peel and Clausen (1982). 22. Craig (1961).

\section{REFERENCES}

Allison, I. F. 1979. The mass budget of the Lambert Glacier drainage basin, Antarctica. Journal of Glaciology, Vol. 22, No. 87, p. 223-35.

Budd, W. F., and Morgan, V. I. 1977. Isotopes, climate, and ice sheet dynamics from core studies on Law Dome, Antarctica. [Union Géodésique et Géophysique Internationale. Association Internationale des Sciences Hydrologiques. Commission des Neiges et Glaces.] Symposium. Isotopes et impuretés dans les neiges et glaces. Actes du colloque de Grenoble, août/septembre 1975, p. 312-21. (IAHS-AISH Publication No. 118.)

Budd, W. F., and Young, N. W. 1979. Results from the I.A.G.P. flow-line study inland of Casey, Wilkes Land, Antarctica. Journal of Glaciology, Vol. 24, No. 90, p. 89-101.

Craig, H. 1961. Standard for reporting concentrations of deuterium and oxygen-18 in natural waters. Science, Vol. 133, No. 3467, p. 1833-34.

Dansgaard, W., and others. 1973. Stable isotope glaciology, by W. Dansgaard, S. J. Johnsen, H. B. Clausen, and N. Gundestrup. Meddelelser om Grønland, Bd. 197, Nr. 2.

Dansgaard, W., and others. 1977. Stable isotope profile through the Ross Ice Shelf at Little America V, Antarctica, [by] W. Dansgaard, S. J. Johnsen, H. B. Clausen, C. U. Hammer, and C. C. Langway, Jr. [Union Géodesique et Géophysique Internationale. Association Internationale des Sciences Hydrologiques. Commission des Neiges et Glaces.] Symposium. Isotopes et impuretes dans les neiges et glaces. Actes du colloque de Grenoble, août/septembre 1975, p. 322-25. (IAHS-AISH Publication No. 118.)

Epstein, S., and Sharp, R. P. 1967. Oxygen- and hydrogen-isotope variations in a firn core, Eights Station, western Antarctica. Journal of Geophysical Research, Vol. 72, No. 22, p. 5595-98.

Epstein S., and others. 1965. Six-year record of oxygen and hydrogen isotope variation in South Pole firn, by S. Epstein, R. P. Sharp, and A. J. Gow. Journal of Geophysical Research, Vol. 70, No. 8, p. 1809-14.

Gonfiantini, R., and others. 1963. Geographical variations of oxygen-18/oxygen-16 ratio in surface snow and ice from Queen Maud Land, Antarctica, by R. Gonfiantini, V. Togliatti, E. Tongiorgi, W. De Breuck, and E. [E.] Picciotto. Nature, Vol. 197, No. 4872, p. 1096-98.

Gordiyenko, F. G., and others. 1976. Variatsii izotopnogo sostava atmosfernykh osadkov i ozernoy vody v Antarktide i Subantarktike [Variations of isotope composition of atmospheric precipitation and lake waters of Antarctica and sub-Antarctica]. [By] F. G. Gordiyenko, N. I. Barkov, A. I. Orlov. Materialy Glyatsiologicheskikh Issledovaniy. Khronika. Obsuzhdeniya, Vyp. 26, p. 150-54.

Johnsen, S. J., and others. 1972. Oxygen isotope profiles through the Antarctic and Greenland ice sheets, |by| S. J. Johnsen, W. Dansgaard, H. B. Clausen, C. C. Langway, Jr. Nature, Vol. 235, No. 5339, p. 429-34; Vol. 236, No. 5344, p. 249.

Katō, K. 1977. Oxygen isotopic composition and gross $\beta$-radioactivity in firn. (In Watanabe, O., ed. Glaciological research program in Mizuho Plateau-west Enderby Land, East Antarctica. Pt. 4, 1974-1975. Tokyo, National Institute of Polar Research, p. 158-69. (Japanese Antarctic Research Expedition. JARE Data Reports, No. 36 (Glaciology).)

Lorius, C., and Merlivat, L. 1977. Distribution of mean surface stable isotopes in East Antarctica: observed changes with depth in the coastal area. [Union Géodésique et Géophysique Internationale. Association Internationale des Sciences Hydrologiques. Commission des Neiges et Glaces.] Symposium. Isotopes et impuretés dans les neiges et glaces. Actes du colloque de Grenoble, aoutt/septembre 1975, p. 127-37. (IAHS-AISH Publication No. 118.) 
Lorius, C., and others. 1969. Variations in the mean deuterium content of precipitation in Antarctica, by C. Lorius, L. Merlivat, and R. Hagemann. Journal of Geophysical Research, Vol. 74, No. 28, p. 7027-31.

Lorius, C., and others. 1979. A 30,000-yr isotope climatic record from Antarctic ice, by C. Lorius, L. Merlivat, J. Jouzel, and M. Pourchet. Nature, Vol. 280, No. 5724, p. 644-48.

Morgan, V. I. 1972. Oxygen isotope evidence for bottom freezing on the Amery Ice Shelf. Nature, Vol. 238, No. 5364, p. 393-94.

Morgan, V. I., and Jacka, T. H. 1981. Mass balance studies in East Antarctica. [Union Géodésique et Géophysique Internationale. Association Internationale des Sciences Hydrologiques.] Sea level, ice, and climatic change. Proceedings of the symposium held 7-8 December 1979 during the 17th general assembly of the International Union of Geodesy and Geophysics, Canberra, p. 253-60. (IAHS Publication No. 131.)

Peel, D. A., and Clausen, H. B. 1982. Oxygen-isotope and total beta-radioactivity measurements on $10 \mathrm{~m}$ ice cores from the Antarctic Peninsula. Journal of Glaciology, Vol. 28, No. 98, p.43-55.

Watanabe, O. 1977. Stratigraphic observations of surface snow cover. (In Watanabe, O., ed. Glaciological research program in Mizuho Plateau - west Enderby Land, East Antarctica. Pt. 4, 1974-1975. Tokyo, National Institute of Polar Research, p. 61-125. (Japanese Antarctic Research Expedition. JARE Data Reports, No. 36 (Glaciology).)

Young, N. W. 1979. Measured velocities of interior East Antarctica and the state of mass balance within the I.A.G.P. area. Journal of Glaciology, Vol. 24, No. 90, p. 77-87. 